\title{
Acknowledgement to Reviewers of Entropy in 2016
}

\section{Entropy Editorial Office}

Published: 11 January 2017

MDPI AG, St. Alban-Anlage 66, 4052 Basel, Switzerland; entropy@mdpi.com

The editors of Entropy would like to express their sincere gratitude to the following reviewers for assessing manuscripts in 2016.

We greatly appreciate the contribution of expert reviewers, which is crucial to the journal's editorial process. We aim to recognize reviewer contributions through several mechanisms, of which the annual publication of reviewer names is one. Reviewers receive a voucher entitling them to a discount on their next MDPI publication and can download a certificate of recognition directly from our submission system. Additionally, reviewers can sign up to the service Publons (https://publons.com) to receive recognition. Of course, in these initiatives we are careful not to compromise reviewer confidentiality. Many reviewers see their work as a voluntary and often unseen part of their role as researchers. We are grateful to the time reviewers donate to our journals and the contribution they make.

If you are interested in becoming a reviewer for Entropy, see the link at the bottom of the webpage http://www.mdpi.com/reviewers.

The following reviewed for Entropy in 2016:

A. Jalab, Hamid

Abah, Obinna

Abbas, Ali E.

Abbas, Ibrahim A.

Abbod, Maysam F.

Abdelaziz, Ramadan

Abdelkefi, Abdessattar

Abe, Sumiyoshi

Abel, Andrew

Abrams, Cameron F.

Abreu, Everton M.C.

Acconcia, Thiago V.

Acharya, U Rajendra

Adami, Chris

Adriaans, Pieter W.

Adrián, Mota-Babiloni

Aggarwal, Vaneet

Ahmadi, Mehdi

Ahmady, Pouria

Ahmed, Mosabber Uddin

Ahn, Choon Ki

Ai, Tinghua

Aiello, Marco

Akih-Kumgeh, Ben

Akritas, Michael

Ala-Nissilä, Tapio

\author{
Alava, Mikko \\ Albarrán, Pedro \\ Albert, Carlo \\ Albert, Victor V. \\ Alicki, Robert \\ Allen, Benjamin \\ Ally, Moonis R. \\ Almeida, Ricardo \\ Alonso, Joan F \\ Álvarez Rodríguez, José María \\ Álvarez-Álvarez, Pedro \\ Amaratunga, Gehan \\ Amelia, Fernanda \\ Amiri, Mehdi \\ Ammon, Martin \\ Andaluz, Joaquín \\ Andersen, Ulrik L. \\ Anderson, Amy D. \\ Andersson, Karin \\ Andrade, Adriano \\ André, José Maria C.S. \\ Andresen, B. \\ Andresen, Bjarne \\ Andris, Clio \\ Aneziris, Olga \\ Anghel, Dragos-Victor
}


Angstmann, C. N.

Angulo, Juan C.

Anishchenko, Lesya

Anna Krawczyk, Dorota

Annibale, Alessia

Annila, Arto

Antucheviciene, Jurgita

Apertet, Yann

Apiecionek, Łukasz

Arabkoohsar, Ahmad

Area, Iván

Arizmendi, Constancio M.

Aronson, Konstantin

Aros, Rodrigo

Arrien, Olatz Ukar

Arroyo, David

Arteche, Josu

Artese, Giuseppe

Arthanari, Tiru

Assad, Syed

Assar, Saïd

Atangana, Abdon

Atkinson, Anthony C

Atmanspacher, Harald

Averin, Dmitri V.

Awad, Mohamed M.

Awan, Zohaib

Azami, Hamed

Azeyanagi, Tatsuo

Azouani, Abderrahim

Azzimonti, Laura

Baba, K.

Baccarani, Giorgio

Back, Juhoon

Badur, J.

Balajewicz, Maciej

Balaji, Bhashyam

Balashov, Victor N.

Balasis, Georgios

Baleanu, Dumitru

Ball, John

Ball, Rowena

Bamba, Kazuharu

Bandt, Christoph

Bang, Jeongho

Baptista, Murilo Da Silva

Barber, Robert

Barbieri, Marco

Barbosa, Ramiro S.

Barbosa, Rui Soares

Barchard, Kimberly

Barletta, Luca
Barnett, Lionel

Barra, Felipe

Barrett, Adam B.

Barrio, Eduardo Alejandro

Basaran, Cemal

Basieva, Irina

Basios, Vasileios

Baskonus, Haci Mehmet

Bassetti, Federico

Basu, Ishita

Batista, G. E. A. P. A.

Bawden, David

Bayram, Suat

Beau, Mathieu

Bedetti, Nicola

Bei, Hongbin

Bell, Bryn

Bellon, Pascal

Belman-Flores, J.M.

Belyaev, Evgeny

Benavoli, Alessio

Benenti, Giuliano

Benninger, Daniel

Benoist, Tristan

Berg, Nathan

Beslon, Guillaume

Bianchini, Cosimo

Bianco, Vincenzo

Billard, Lynne

Bizarro, João P. S.

Blachnik, Marcin

Blakely, Jonathan N.

Blanc, Philippe

Blasco, Jorge

Bohannan, Gary

Bojowald, Martin

Bondarescu, Ruxandra

Boos, Dennis D.

Bordoni, Stefano

Borg, Markus

Borghesani, Pietro

Borodich, Feodor M.

Bossomaier, Terry

Boström, Kim

Boudraa, Abdel-Ouahab

Bouju, Alain

Bountis, Tassos

Bourgoin, Jean-Philippe

Boyer, Timothy

Boyom, Michel

Boyom, Michel Nguiffo

Branciard, Cyril 
Brandner, Kay

Braun, Dieter

Brewer, Brendon

Broadbridge, Philip

Brooks, Richard

Brooks, Richard R.

Broom, Mark

Brownlee, Sandy

Bruschi, David Edward

Budroni, Costantino

Buntine, Wray

Burbano, Daniel

Busa, Michael A.

Buscemi, Francesco

Cabal-Yepez, Eduardo

Cabboi, Alessandro

Cabral, João M. G.

Cabral, Pedro

Cacace, Marcello G.

Cacciatori, Sergio L.

Caceres, Elena

Cafaro, Carlo

Calcagnì, Antonio

Calero Valdez, André

Calvo De Lara, José Ramón

Calvo Hernandez, Antonio

Campa, Alessandro

Cancho, Ramon Ferrer i

Cannella, Salvatore

Canovas Pena, Jose S.

Caponetto, Riccardo

Capraro, Valerio

Caravagna, Giulio

Caravelli, Francesco

Carbone, Anna

Carlini, Maurizio

Casadio, Roberto

Casarin, Roberto

Cassetti, Gabriele

Castaños, Octavio

Castro, Enrique

Castro, Mauricio

Catarino, Paula M.C.

Cavada, María Ortiz

Cavalcanti, Eric

Cavalli, Fausto

Cembranos, J. A. R.

Cerbino, Roberto

Cerda, Mauricio

Cerulo, Luigi

Cespedes, Patricia Conde

Cetto, Ana Maria
Chacón-Acosta, Guillermo

Chaiken, Seth

Chakraborty, Biman

Chalishajar, Dimplekumar

Chamberlin, Ralph

Chambers, Jonathon

Chang, Zheng

Chau, Yuen

Chejne, Farid

Chen, Bor-Sen

Chen, Chien-Chung

Chen, Fu-Hsiang

Chen, GuoQian

Chen, Hanshuang

Chen, Jr-shian

Chen, Kuan-Yu

Chen, Lin

Chen, Shyi-Ming

Chen, Ting-li

Chen, Wei-shing

Chen, Yu-Bin

Chen, Yuhua

Chen, Zhiyuan

Cheng, Chen-Yang

Chesneau, Xavier

Chiavazzo, Eliodoro

Chiementin, Xavier

Chirikjian, Gregory S.

Chiu, Ming-Chuan

Choi, Young-Seok

Christen, Thomas

Chryssolouris, George

$\mathrm{Chu}$, Hai-Cheng

Chung, Kung-Ming

Ciavolino, Enrico

Cieliebak, Mark

Claesson, Per Martin

Clarke, Tomas G.

Clement, Simon

Cluni, Federico

Codetta-Raiteri, Daniele

Colangelo, Gianpiero

Collet, Philippe

Comez, Lucia

Comi, Antonio

Concezzi, Moreno

Condoluci, Massimo

Conte, Elio

Cools, Mario

Corominas-Murtra, Bernat

Corsini, Alessandro

Costa, V.A.F. 
Costola, Michele

Couillet, Romain

Craciun, Georghe

Crepeau, John

Croce, Giulio

Croke, Sarah

Cruces, Sergio

Cruz-Hernández, César

Cuesta-Frau, David

Cui, Huijuan

Cummins, Fred

Cushman, Samuel A.

D'Abramo, Germano

D'Agostino, Gregorio

Dahlsten, Oscar

Dai, Zhendong

Daivis, Peter J.

Dall'Asta, Luca

Damasevicius, Robertas

D'Ambrosio, Vincenzo

Danehkar, Ashkbiz

Daniusevičiute, Laura

Das, Buddhadeva

Das, Saurya

Datcu, Mihai

Datta, Soumya Kanti

Davies, Paul

Davis, Roger

Davison, Matt

Dazzi, Patrizio

De Amorim, Renato Cordeiro

de Araujo, Jose Carlos

De Bragança Pereira, Carlos Alberto

De Brevern, Alexander

De Campos, Cassio P.

De Chiara, Gabriele

De Coninck, Joël

De Felice, Fabio

De Los Rios, Paolo

De Oliveira Junior, Silvio

De Oro Calderon, Raquel

De Pascale, Andrea

De Rosis, Alessandro

De Sortis, Adriano

De Valcárcel, Germán J.

Debowski, Lukasz

DeDeo, Simon

Deffner, Sebastian

Del Campo, Adolfo

Del Jesus Díaz, Maria Jose

D'elia Branco, Márcia

Delvenne, Jean-Charles
Demarie, Tommaso

Demidenko, Eugene

Deng, Fu-guo

Der, Ralf

Destercke, Sébastien

Destrempes, François

Dewil, Raf

Di Gravio, Giulio

Di Crescenzo, Antonio

Di Giulio, Massimo

Di Lena, Pietro

Di Lorenzo, Antonio

Di Vita, A.

Diana, Giovanni

Dias, Claudia Camila

Dickhaus, Thorsten

Diks, Cees

Dinh, TN

Dobre, Ciprian

Dohnal, Vlastislav

Dolan, Brian

Dologlou, Yannis

Dong, Yuan

Donner, Reik

Dorić, Jovan

Drazer, German

Drigo Filho, Elso

Drikakis, Dimitris

Dritselis, Chris

Drovandi, Christopher C.

Du, Weiwei

Duarte-Mermoud, Manuel A.

Dubbeldam, Johan

Dukes, Peter J.

Dulf, Eva H.

Duque, Carlos

Dvali, Georgi

Dwivedi, Dipankar

Dziarmaga, Jacek

Echevarría, Miguel G.

Eduardo Virgilio da Silva, Luiz

Eguiraun, Harkaitz

Eidsvik, Jo

Elechum, Hichem

Eleuch, Hichem

El-Khazali, Reyad

Ellahi, Rahmat

Ellahi, Rahmat

Ellinas, Demosthenes

Elmegaard, Brian

Eloe, Paul W

El-Sayed, Ahmed 
El-Sayed Ali, Mohamed

Elwakil, Ahmed S.

Emran, Khadijah M.

Enteria, Napoleon

Epinat, Benoît

Escassut, Alain

Esposito, Massimiliano

Estellé, Patrice

Esteves, Luís Gustavo

Evans, Michael

Evans, Robin

F. Sanjuan, Miguel Á.

Fabijanska, Anna

Fabrizio, Mauro

Faes, Luca

Faggini, Marisa

Falniowski, Fryderyk

Fang, Hui

Faraut, Jacques

FATU, Aurélian

Federolf, Peter

FEIDT, Michel

Feixas, Ferran

Felice, Domenico

Feng, Yu

Fera, Marcello

Fernández, Juan José

Fernández, Nelson

Fernández Bariviera, Aurelio

Ferrara, Massimiliano

Ferrari, Carlo

Ferreira, Flávio

Ferreira, Paulo

Ficco, Massimo

Fidaleo, Francesco

Figliola, Alejandra M.

Figlus, Tomasz

Figueiredo, Lino

Figueras, $\mathrm{Pau}$

Figueroa, Aldo

Fijalkow, Inbar

Filatrella, Giovanni

Fillion-Gourdeau, François

Fischer, Asja

Fischer, Richard

Fistola, Romano

Fleury, Martin

Flindt, Christian

Florens, Serge

Flores, Juan J.

Fogolari, Federico

Fortuna, Luigi
Foti, Dora

Frances-Villora, Jose Vicente

Frank, Steven

Frauchiger, Daniela

Freeborn, Todd J.

Frenkel, Sergey

Frey, Erwin

Friston, Karl

Frolov, Valeri

Fronsdal, Christian

$\mathrm{Fu}$, Gengshen

Fuentes, Miguel

Fuller, Steve

Fung, Benjamin C. M.

Fung, Thomas

Gabarda, Salvador

Gadomski, A.

Gadouleau, Maximilien

Gaggioli, Richard A.

Galhano, Alexandra

Gallas, Jason

Gallavotti, Giovanni

Gallegati, Marco

Galperin, Michael

Galperine, I.

Gamo, Javier

Gao, Chen

Gao, Lei

Gao, Michael

Gao, Song

Gao, Zhongke

Garcia-Perez, Arturo

Gardini, Laura

Gay-Balmaz, Francois

Gazeau, Jean Pierre

$\mathrm{Ge}, \mathrm{Hao}$

Geiger, Bernhard

Gelbwaser, David

Gen Cai, Rong

Gencaga, Deniz

Gençay, Ramazan

Gendron, Paul

Genes, Claudiu

Georgiou, Emmanuel

Georgoulas, George

Gerard, Leng

Gershenson, Carlos

Gharari, Shervan

Gharibian, Sevag

Ghoraani, Behnaz

Ghosh, Bidisha

Ghosh, Joydip 
Ghosh, Kingshuk

Giannerini, Simone

Giannoccaro, Ilaria

Gibaja, Eva

Giffin, Adom

Ginsca, Alexandru Lucian

Giorgi, Gianluca

Giovanni, Fusco

Gludovatz, Bernd

Goebel, Rafal

Goeman, Jelle

Goes, Luiz Carlos Sandoval

Goggans, Paul

Gogolin, Christian

Goicochea, A. Gama

Goldman, Jennifer Sarah

Goldschmidt, Elizabeth

Goldstein, Markus

Gómez, Luis

Gomez-Aguilar, J.F.

Gomez-Barrero, Marta

Gonçalves, Hernâni

Gong, Jiangbin

Gong, Mei

González, Javier

González-de-la-Rosa, J. J.

Gonzalez-Lopez, V. A.

González-Martel, Christian

Goparaju, Sreechakra

Gorban, Alexander

Gorji-Bandpy, Mofid

Goshtasby, A. Ardeshir

Gozzi, Ennio

Grabowski, Janusz

Gradoni, Gabriele

Gramacy, Robert B.

Grammenos, T.

Grassi, Antonio

Grau, Jan

Greig, Alistair

Grendar, Marian

Grieve, Stuart W. D.

Grigonyte, Gintare

Grillakis, Manolis

Grinbaum, Alexei

Gritli, Yasser

Grmela, Miroslav

Grøn, Øyvind

Grothe, Oliver

Grzymala-Busse, Jerzy W.

$\mathrm{Gu}$, Fengshou

Guariglia, Emanuel
Guccione, Pietro

Guedes, I.

Guillaume, Joseph

Guoxing, Lin

Gutierrez Rojas, H. A.

Gutiérrez Tobal, Gonzalo C.

Hadad, Yossi

Hall, Ken

Hall, Michael

Hameed, Muhammad

Hamza, A. Ben

Hand, David

Hanfelt, John

Hangos, Katalin

Hanriot, Sérgio De Morais

Hans Jürgen, Korsch

Haq, Rizwan

Hariri-Ardebili, Mohammad Amin

Harko, Tiberiu

Harnefors, Lennart

Harremoës, Peter

Harrison, Willie

Harshman, Nathan

Hasegawa, Takehisa

Hashizume, Hiromichi

Hashizume, Yoichiro

Hayashida, Morihiro

Hayter, Anthony J.

He, Jihuan

He, Kaijian

$\mathrm{He}$, Ran

He, Yurong

Heidelmann, Markus

Heidrich-Meisner, Fabian

Helayël-Neto, José Abdalla

Henard, Christopher

Henchman, Richard

Henebry, Geoffrey

Hennigar, Robie A.

Hermes, Christian J. L.

Herrmann-Pillath, Carsten

Herwig, Heinz

Hetet, Jean-Francois

Hidalgo, Arturo

Hinkle, Jacob

Hlavac, Vaclav

Hobbie, Erik K.

Hoeller, Yvonne

Hohenwarter, Anton

Holden, Sean B

Holland, Daniel J.

Holm, Elizabeth A. 
Holobar, Aleš

Holubec, Viktor

Holzinger, Andreas

Hong, Keum-Shik

Hong, Wei-Chiang

Hong, Wenjing

Honig, Jurgen

Horgnies, Matthieu

Horsch, Martin

Hosni, Hykel

Hou, Shuhn-Shyurng

Hovhannisyan, Karen

Hribernik, Ales

Hrissanthou, Vlassios

Hsiao, Bo

Hsiao, Kai-Long

Hsu, Ming-wei

$\mathrm{Hu}$, Feng

$\mathrm{Hu}$, Ya-Peng

$\mathrm{Hu}$, Yuening

Huang, Can

Huang, E-wen

Huang, Ke

Huang, Lucy

Huang, Minlie

Huang, Shenwei

Huang, Weimin

Huang, Wen

Huatuco, Luisa

Huber, Marcus

Hughes, Ifan

Hughes, Keith H.

Huminic, Angel

Huminic, Gabriela

Hung, Ling-Yan

Hutter, Marcus

Huveneers, François

Ibañez, Guillermo

Ichiki, Kiyotomo

Ide, Yusuke

Idier, Jérôme

Ikuta, Rikizo

Ilg, Patrick

Ilia, Ioanna

Ilić, Aleksandar

Iliyasu, Abdullah M.

Inard, Christian

Ionescu, Valeriu Manuel

Iosifidis, Alexandros

Irpino, Antonio

Isidro, Jose M.

Islam, Shareeful
Ito, Sosuke

Itoh, Mitsuhiro

Izbicki, Rafael

Izquierdo, Joaquim Blesa

Jabbarzadeh, Ahmad

Jacinto, Carlos Magno Couto

James, Ryan G.

Jameson, Geoff

Jammalamadaka, S. R.

Jammeh, Emmanuel

Jang, Gun Ho

Jarohs, Sven

Jasra, Ajay

Jauregui, Juan Carlos

Jelinek, Herbert F.

Jia, Junbo

Jiang, Liangxiao

Jiang, Peixue

Jiang, Xi

Jiménez, Antonio

Jin, Ningde

Jin, Yan

Jordan, Andrew N.

Joshi, Chaitanya

Joshi, Karuna Pande

Jun, Sunghae

Jupp, Peter

Jus, Kocijan

Kabiraj, Parijat

Kahirdeh, Ali

Kaj, Ingemar

Kalogerias, Dionysios

Kalosakas, George

Kamiński, Marcin

Kamsu-Foguem, Bernard

Kan, Xingye

Kanamori, Takafumi

Kaneta, Motohiro

Kang, Kyongok

Kang, Myeongsu

Kang, Sungwon

Kaniadakis, Giorgio

Kannan, Ramakrishnan

Kapadia, Mubbasir

Karch, Andreas

Karczmarek, Paweł

Kargol, Armin

Karimi, Nader

Karmakar, Chandan Kumar

Karpov, Evgueni

Kasaeian, Alibakhsh

Kasser, Jeffrey 
Kassubek, Frank

Kastner, Ruth

Kato, Kentaro

Katriel, Jacob

Katsavounidis, Ioannis

Kaufman, Adam

Kaufman, Michael

Kawazura, Yohei

Keller, Karsten

Kelly, Wayne A.

Kemp-Benedict, Eric

Kenett, Dror Y.

Kerr, David

Keßler, Carsten

Khonsari, Michael

Khoshmanesh, Khashayar

Kiermaier, Michael

Kiessé, Tristan Senga

Kihl, Maria

Kim, Eun-Ah

Kim, Harksoo

Kim, Min Soo

Kim, Taekyun

Kivelä, Mikko

Klados, Manousos

Kleer, Robin

Kleinmann, Matthias

Klimenko, Alexander Y.

Knezevic, Irena

Knight, Charles

Knuth, Kevin

Ko, Chia-Nan

Koch, Eckhard

Kohar, Vivek

Kokjohn, Sage

Kolev, Nikolai Valtchev

Kolkiewicz, Adam W.

Kondepudi, Dilip K.

Kontoyiannis, Ioannis

Kordos, Mirosław

Kosta, Sokol

Kostowski, Wojciech

Kotsiantis, Sotiris

Kotyra, Andrzej

Kozyrev, Sergey

Krajsek, Kai

Kraniotis, G. V.

Krestel, Ralf

Kroiss, Alexander

Krüger, Björn

Krzysztofik, Wojciech J.

$\mathrm{Ku}$, Lun-Wei
Kugiumtzis, Dimitris

Kulikova, Maria

Kumar, Pardeep

Kumar, S. Chaitanya

Kumar, Sajeesh

Kumar, Suren

Kurniawan, Michael

Kurowicka, Dorota

Kurz, Sascha

Kustova, Elena

Kwietniak, Dominik

Lacroix, Yves

Lake, Douglas E.

Laming, Donald Richard John

Lampart, Marek

Lawrence, Albert

Lê, Hông Vân

Le Clainche, Soledad

Lee, Deokjung

Lee, Hee Jin

Lee, Moo-Yeon

Lee, $S$.

Lee, Sun-Kyu

Leff, Harvey

Leff, Harvey S

Lehmann, Christopher MB

Lei, Sun

Lei, YaGuo

Leibovici, Didier

Lelea, Dorin

Lentmaier, Michael

Lenzi, Ervin K.

Lenzi, Ervin Kaminski

Leonski, Wiealaw

Lepe, Samuel

LePoire, David

Lerner, Vladimir S.

Lesenfants, Damien

Leugering, Günter

Levy, Matthew Chase

Li, Bai-Lian

Li, Cheng-Hsuan

Li, Der-Chiang

Li, Duan

Li, Guanchen

Li, Ming

Li, Tiancheng

Li, Xiaohua(Edward)

Li, Xiaoli

Li, Yingsong

Liang, Hualou

Liang, Yu 
Liao, Hung-chang

Licata, Ignazio

Lien, Chang-Hua

Lijffijt, Jefrey

Lim, Charles Ci Wen

Lin, Pei-Feng

Lin, Shih-Chun

Lin, Yu-Jau

Lineweaver, Charles

Lipponen, Jukka A.

Liskiewicz, Tomasz

Liu, Han

Liu, Jianquan

Liu, Jie

Liu, Lanbo

Liu, Yimin

Liu, Yuan

Liu, Zhigang

Lo Storto, Corrado

Lombardi, Olimpia

Long, Guilu

Longo, Sandro

Lopez-de-Ipina, Karmele

Löser, Wolfgang

Lostado, Ruben

Loukas, George

Lu, Tsung-Hsun

Lubczonek, Jacek

Luchko, Yuri

Lucia, Umberto

Luhandjula, M.K.

Lunin, Oleg

Luongo, Orlando

Lupo, Cosmo

Lutz, Eric

Luukka, Pasi

Lynn, Theo

Łyszkowski, Radosław

M. Martyushev, Leonid

Ma, Duancheng

Ma, Hui

Ma, Jinwen

Ma, Tinghuai

Ma, Xiaoli

Maas, Axel

Machado, J. A. Tenreiro

Machado, Jose

Machrafi, Hatim

Maciejewski, Wesley

Mady, Carlos E.k.

Magnus, Ryden

Mahdavi, Mahboobe
Mahian, Omid

Mahmoudi-Larimi, Yasser

Mahmud, Shohel

Makinde, O.D.

Maluckov, Aleksandra

Mamede, Jorge

Mammone, Nadia

Mancini, Stefano

Mandal, Dibyendu

Mandic, Danilo

Maniu, Silviu

Mann, Robert B.

Manta, Vasile I.

Manzanares, José Antonio

Manzoni, Anna M.

Mao, Xiaoan

Marchesoni, Fabio

Marcon, Eric

Marek-Crnjac, Leila

Mares, Edwin

Marini, Gustavo

Mark, Kedzierski

Markowski, Konrad Andrzej

Marmur, Abraham

Marreiros, Goreti

Marrero, Juan Carlos

Martin, Michael J.

Martín, David

Martinelli, Mario

Martínez Álvarez, Francisco

Martins, Bruno

Martins, Christiane Frigerio

Martyushev, L.M.

Mashima, Daisuke

Maskell, Simon

Masmoudi, Atef

Mastroberardino, Antonio

Matei, Sorin Adam

Mathias, Mauro Hugo

Matsoukas, Themis

Maundy, Brent

Mauri, Roberto

Mauris, Gilles

Mayer, Christopher

Mazurek, Przemysław

McBride, Carl

McCracken, Richard A.

McKay, Brendan

Mckay, Brendan D.

McKenzie, Grant

McPherson, N. A.

Medjroubi, W. 
Meher, Pramod Kumar

Meiburger, Kristen M.

Mejia-Monasterio, Carlos

Melia, Umberto

Melício, Rui

Melucci, Massimo

Méndez, Federico

Menezes, Pradeep L.

Meo, Marianna

Meo, Pasquale De

Mercorelli, Paolo

Mertens, Thomas G.

Mesbah, Ali

Meyerovitch, Tom

Mezei, József

Micalizio, Salvatore

Micheli, Alessio

Michelitsch, Thomas M.

Michor, Peter

Mien, Van

Miguel, Antonio Ferreira

Mikhelson, Ilya V.

Miłkowski, Marcin

Miller, Casey W.

Milne, Bruce

Minami, Ichiro

Ming, Dengming

Minnhagen, Petter

Minussi, Carlos Roberto

Miret-Artés, Salvador

Misiurewicz, Michal

Modak, Sujoy K.

Modrak, Vladimir

Mohammadpour, Hossein Ali

Molica Bisci, Giovanni

Molinaroli, Luca

Molina-Vilaplana, Javier

Mondejar, Maria E.

Montrucchio, Bartolomeo

Moonis, Majaz

Morabito, Francesco Carlo

Moran, Bill

Morandi, Omar

Moraru, Luminița

Moreno, Hilario Gómez

Morgado, Welles Antonio Martinez

Morioka, Yushi

Morison, Gordon

Morita, Kazuki

Morita, Takeshi

Morosuk, Tatiana

Moroz, Adam
Mortazavi, Vahid

Moscoso Del Prado Martin, Fermín

Mosk, Allard

Mota, Alzira

Mota, David

Moustakidis, Charalampos

Mowshowitz, Abbe

Mporas, Iosif

Mück, Wolfgang

Muga, Gonzalo

Muhammad Belgacem, Fethi

Mulder, Joris

Müller, Markus P.

Müller, Sebastian

Murtagh, Fionn

Muscato, Orazio

Musgrave, Ian

Musio, Monica

Myers, John M.

Nagase, Takeshi

Naik, Ganesh R.

Nanni, Loris

Naseer, Noman

Naser, Jamal

Nash, David

Naudts, Jan

Navarro, Jorge

Nechita, Ion

Negrel, Romain

Neta, Beny

Newman, Kimberly E.

$\mathrm{Ng}$, Jason

$\mathrm{Ng}$, Wee Siong

Nguyen, Xuan Vinh

Nicolaou, Nicoletta

Nieto, Juan J.

Nieuwenhuizen, Theo

Nikas, George K.

Nikolaou, Konstantinos

Nikulchev, Evgeny

Nikulov, Alexey

Niven, Robert

Noble, Denis

Nobre, Fernando D.

Noro, Marco

Nosonovsky, Michae

Novaes, Marcel

Nowman, Ben

Nygård, Kim

Ocaña, Jordi

Odintsov, Sergei

Ogiela, Marek 
Oh, Hyondong

Oikonomou, Thomas

Oikonomou, V.K.

Oliveira, José Valente De

Oliveira, Paulo Eduardo

Oliveira, Santiago Del Rio

Ollivier, Yann

Olmo, Gonzalo J.

Olmos, Pablo M.

Olson, Michael W.

Ommen, Torben

Opatrný, Tomás

Oprea, Iuliana

Ormos, Mihály

Oryshchenko, Vitaliy

Oshanin, Gleb

Otero, Cesar

Oyabu, Takeshi

Ozaktas, Haldun M.

Özarslan, Mehmet Ali

Ozcan, Hasan

Ozturk, Erdem

Paganoni, Anna Maria

Pagliarini, Giorgio

Paja, Wieslaw

Palao, José P.

Palestini, Arsen

Palomares, Iván

Palumbo, D.

Pang, Yi

Paoluzzi, Matteo

Papadimitriou, Ioannis

Papadopoulos, Symeon

Papadopoulos, Yiannis

Papi, Serena

Papic, Zlatko

Park, J.

Park, Jin Han

Park, K. R.

Parraguez, $\mathrm{P}$.

Parry, Matthew

Pastore, Raffaele

Patel, Raj

Patera, Giuseppe

Paternostro, Mauro

Patriota, Alexandre Galvão

Paul, Biplab

Paul, Titan C.

Pauluis, Olivier M.

Pavón, Diego

Payaró, Miquel

Payne, William V.
Pecina, Pavel

Pedraza, Juan F.

Peharz, Robert

Pejaś, Jerzy

Pelster, Axel

Pennini, F.

Perc, Matjaz

Peres, David J.

Perezgonzalez, Jose

Peron, Thomas

Pescapè, Antonio

Pessa, Eliano

Peter, Adrian M.

Peterson, Tim

Petz, Gerald

Peyrodie, Laurent

Pfante, Oliver

Pfenniger, Daniel

Pham, Tuan D.

Phinyomark, Angkoon

Piacentino, Antonio

Piantadosi, Steven T.

Pickering, Ed

Pigolotti, Simone

Pinchas, Monika

Pincus, David

Pineiro, Manuel M.

Pinho, Armando

Pinto, Carla

Pinto, Carla M.A.

Pires, Victor

Pistone, Giovanni

Planat, Michel

Plastina, Francesco

Plastino, Angel Ricardo

Plastino, Angelo

Pleimling, Michel

Pohl, Christine

Poletti, Marco Gabriele

Polettini, Matteo

Polizzotto, Nicola R.

Polo, Jose Luis

Ponsoda, Enrique

Poole, Charles

Pop, Ioan

Popescu, Sandu

Porta, Alberto

Poti, Valerio

Potirakis, Stelios

Povstenko, Yuriy

Pradas, Marc

Pradeep, Konda 
Pradeep, Konda Gokuldoss

Preißinger, Markus

Prussenar, Gunnar

Psannis, Konstantinos

Psarakis, Stelios

Psychalinos, Costas

Pustelnik, Nelly

Puthoff, H. E.

Qiu, Jing

Qu, Guangzhi

Qu, Zhongjun

Quang, Minh Ha

Quarati, Pierino

Quarati, Piero

Quessy, Jean-François

Rabinovici, Raul

Radcliffe, Mary

Radi, Davide

Raginsky, Maxim

Raimundo, Joana

Rajendra Acharya, U.

Ramachandran, K. I.

Ramirez, Pedro E.

Ramos, Rubens Viana

Ramos, Victoria

Rangaiah, G.P.

Rangel, Victor

Rao, Prahalad K

Rao, Shankar

Raspet, Richard

Rathore, Saima

Ratiu, Tudor

Rauh, Johannes

Razavi, Mohsen

Reades, Jonathan

Reboredo, Juan C.

Reddy, Yenumula

Reeb, David

Remeseiro, Beatriz

Ren, Tsang Ing

Rennie, Frank

Requardt, Manfred

Rex, Andrew F.

Reygner, Julien

Rezaei, Jafar

Rezakhani, Ali

Ribeiro, Haroldo Valentin

Ribeiro, Ricardo

Ricciuti, Costantino

Richardson, E.S.

Rienow, Andreas

Rigolin, Gustavo
Rimini, Bianca

Rinaldi, Fabio

Rinaldi, Massimiliano

Ritchie, Mark E.

Roberts, David W.

Robinson, Tom

Rocca, M. C.

Rocchetti, Andrea

Rocha, J. Leonel

Rocha, Jorge V.

Rodrigues, Alexandre A. P.

Rodrigues, Clóves G.

Rodrigues, Francisco A.

Rodríguez, Juan M. C.

Roe, Philip L.

Roeck, Wojciech

Rogatko, Marek

Rogers, David

Rogosin, Sergei

Roldán, Édgar

Romano, Elvira

Romanos, Panayiotis

Romero, Ric

Roscia, Mariacristina

Rosenhaus, Vladimir

Rossi, Luca

Roughan, Matthew

Roumeliotis, Ioannis

Rovetta, Stefano

Rozmus, Wojciech

Ruano, António

Rubi, J. Miguel

Rubio, F. Javier

Rugh, Hans Henrik

Ruiz-Cárcel, Cristobal

Runge, Jakob

Ruppeiner, George

Ruzzenenti, Franco

Rychtar, Jan

Sabín, Carlos

Sadiki, Amsini

Sadinle, Mauricio

Safari, Mehdi

Sagar, Robin P.

Saha, U.K.

Sahidullah, Md

Sakajo, Takashi

Salata, Ferdinando

Samdberg, Frida

Sánchez Ruiz, Luis Manuel

Sanchis, Manuel

Sanders, Jason 
Sandoval, Leonidas

Sands, David

Sandstad, Marit

Santafé, Guzmán

Santodonato, Lou

Santos, Andres

Santos, Jorge E.

Santos, Lea F.

Sanz-Alonso, Daniel

Saracoğlu, Bayındır H.

Sarbu, Ioan

Sardellitti, Stefania

Saridakis, Emmanuel N.

Sasso, Maurizio

Sauveron, Damien

Saxena, Navrati

Sayed-Mouchaweh, Moamar

Scala, Antonio

Scarfone, Antonio M.

Schaefer, Rafael F.

Schaeffer, Satu Elisa

Schaller, Gernot

Schieber, Jay

Schlegel, Michael

Schmandt, Bastian

Schmelzer, Jürn W.P.

Schmidt, Christian Andrés

Schmitt, François G.

Schmitt, R.H.

Schneider, Michael D.

Schraudner, Michael

Schroeder, Daniel V.

Schuch, Dieter

Schuerrer, Ferdinand

Schulman, Larry

Schwander, Olivier

Schwarz, D. J.

Sciubba, Enrico

Sechidis, Konstantinos

Seghouane, Karim

Segnon, Mawuli

Segundo-Ramírez, Juan

Sembajwe, Grace

Senabre, C.

Senkov, Oleg

Sergiou, Charalambos

Serra, Luis

Seshadreesan, Kaushik

Seward, Brandon

Sewell, Geoffrey

Shamim, Tariq

Shang, Yilun
Shanmugam, Ram

Sheehan, D. P.

Shen, Alexander

Shen, Shengping

Sheng, Guo

Sheremet, Mikhail

Sheremet, Mikhail A.

Shi, Peng

Shi, Yong

Shi, Yongtang

Shiaeles, Stavros

Shibata, Hiroyuki

Shikano, Yutaka

Shin, Yong-June

Shinohara, Russell Takeshi

Shojafar, Mohammad

Shutters, Shade T.

Siedl, Christoph

Sieniutycz, Stanislaw

Sikora, Jan

Simani, Silvio

Simon, Ann

Simon, Joan

Singleton, Douglas

Sitányiová, Dana

Skotiniotis, Michalis

Slussarenko, Sergei

Small, Michael

Śmieja, Marek

Smith, Edward R.

Smith, Eric

Snoke, D.w.

Soares, Maria Joana

Soares-Pinto, Diogo O.

Sodini, Mauro

Solteiro Pires, Eduardo José

Soltys, Michael

Som, Subhojit

Sommer, Stefan

Song, Chunqiao

Sookhak, Mehdi

Sordoni, Alessandro

Soriano, Miguel C.

Sothmann, Björn

Sousa-Silva, Rui

Soylu, Ahmet

Sparavigna, Amelia Carolina

Stachurski, Zbigniew $\mathrm{H}$.

Staiano, Antonino

Stavroulakis, Ioannis P.

Stefanatos, Dionisis

Stekler, Herman 
Stepanov, Nikita D. Stern, Julio Michael Stevenson, Robert Stich, Michael Stoean, Catalin Stojkovic, Dejan Stonaha, Paul J. Stouffs, Pascal Stramaglia, Sebastiano Strozzi, Fernanda Štys, Dalibor Subiantoro, Alison Sun, Caizhi Sun, Jiangwen Sun, Lei Sushko, Iryna Svozil, Karl Swapna, Gottipati Swendsen, Robert Syta, Arkadiusz Szabo, Lorand Szczepanski, Janusz Szigeti, Stuart Szpyrka, Marcin Tabrizi, Ali Akbar

Taddei, Fabio

Tadmor, Rafael Tadokoro, Yukihiro Takao, Manabu Tan, Chung-I Tandon, Rashish Tanimoto, Jun Tawfik, Abdel Nasser Teerikorpi, Pekka Temko, Andriy Tempesti, Duccio Tenne, Reshef Teo, Edward Teodorescu, Iuliana Teodorescu, Razvan Teplyakov, Andrew V. Thalhammer, Mechthild Tiakas, Eleftherios Tian, Fuyang Tian, Guohong Tian, Zhen F. Tieve, Hélio Timme, Nicholas Tirkel, Andrew Tlili, Iskander Tohme, Fernando Tolias, Giorgos
Toma, Cristian

Tomai, Eleni

Tomar, Vikrant Singh

Toporensky, Aleksey

Torabi, Mohsen

Toreyin, Behcet Ugur

Torre, Renato

Torres, Ricardo Da S.

Torres Cebrian, Abel

Tous, Ruben

Tovstolis, Alexander

Tramontana, Fabio

Treffinger, Peter

Trépanier, Jean-Yves

Triantaphyllou, Evangelos

Trivellato, Barbara

Trovati, Marcello

Tsagarakis, Konstantinos

Tsai, Chun-Wei

Tsai, Yao-Hong

Tsallis, Constantino

Tsang, Kim Fung

Tscharner, Vincent Von

Tseng, Chih-Yuan

Tsonis, Anastasios

Tsvetkov, Pavel

Tsyganova, Julia V.

Tuck, Adrian

Tufarelli, Tommaso

Tura, Jordi

Turalska, Malgorzata

Türkyılmazoğlu, Mustafa

Twining, Carole

Tzirtzilakis, E.

Uffink, Jos

Uhrig, Götz S.

Ulanowicz, Robert

Uohashi, Keiko

Usenko, Vladyslav

Vahdati, Mehdi

Vakkilainen, Esa

Valdinoci, Enrico

Valenti, Davide

Valenzuela-Valdés, Juan F.

Valério, Duarte

Valero, Antonio

Valverde, Sergi

Ván, Peter

Van Baalen, Minus

Van De Vis, Hans

Van Vliet, Marijn

Vandenberghe, Lieven 
Varga, Lajos Károly

Vasan, Arvind

Vasquez Padilla, Ricardo

Velten, H. E. S.

Venegas, Maria

Ventura, Sebastián

Verdoolaege, Geert

Verrucchi, Paola

Vianna, Reinaldo Oliveira

Vicente, Renato

Villani, Marco

Villecco, Francesco

Villena, Marcelo J.

Vilone, Daniele

Vlachos, Nicholas

Vladimirov, Andrei

Vogel, Carl

Volkov, Alexander G.

Volos, Christos

Von Toussaint, Udo

Von Tscharner, Vinzenz R.

Vourdas, Apostol

Vowinckel, Bernhard

Waegeman, Willem

Wagner, Sebastian

Waldner, Peter

Walk, Nathan

Walter, Gero

Wang, Anzhong

Wang, Gai-Ge

Wang, Hua

Wang, Kevin

Wang, Xikui

Wang, Xingyuan

Wang, Xun

Wang, Zhu

Warrens, Matthijs J.

Warwicker, Jim

Wasserstein, Ronald L.

Watanabe, Eiichi

Waterstraat, Nils

Waytowich, Nicholas

Weaver, Mark

Wei, Shuangqing

Weichhart, Georg

Weidmann, Claudio

Weinberg, Graham V

Weir, Bruce

Weitzen, Jay A.

Wellek, Stefan

Weng, Huei Chu

Wenger, Urs
Wessel, Niels

West, Bruce J.

White, Roger

Whitney, Robert

Whittaker, Joe

Wichert, Andreas

Wicks, Michael

Wierzbicki, Michał

Wiesner, Karoline

Wilhelm-Mauch, Frank

Wilson, Christopher G.

Witkowski, Alfred

Wiuf, Carsten

Wong, Ka-Chun

Wong, Kon Max

Wong, Shwin-Chung

Wood, Mitchell

Wootters, William

Wright, Jon

Wu, Chih-Hang

$\mathrm{Wu}$, Chung-min

$\mathrm{Wu}$, Hsien-Tsai

Wu, Junde

Wu, Lifeng

Wu, Qiang

$\mathrm{Wu}$, Shuen-De

Wu, Tian-Yau

$\mathrm{Wu}$, Xinjun

Würtz, Rolf P.

Wyse, Jason

Xiao, Boqi

$\mathrm{Xu}$, Mingtian

$\mathrm{Xu}$, Yan

Yablonsky, Gregory

Yagi, Hideki

Yamano, Takuya

Yan, Xinggang

Yang, Can

Yang, Min-Hsiung

Yang, Thomas T

Yang, Wen

Yang, Yong

Yang, Zhi-Xin

Yao, Kai

Yasuda, Muneki

Yeh, Jien-Wei

Yeo, Junyeob

Yger, Florian

Yi, Sang-Hoon

Yin, Liping

Yoneyama, Jun

Young, Derek S. 
Yu, Meilin

Yuen, Ka-Veng

Yun, Rin

Zanardi, Paolo

Zanin, Massimiliano

Zatar, Wael

Zavadskas, Edmundas K.

Zbinden, Hugo

Zeeshan, Ahmad

Zenil, Hector

Zhan, Lingwei

Zhang, Aidong

Zhang, Baohua

Zhang, Haijun

Zhang, Hai-Qing

Zhang, Hao

Zhang, Hongguang

Zhang, Jie

Zhang, Jun

Zhang, Lei

Zhang, Liping

Zhang, Wenming

Zhang, Xufang
Zhang, Ya

Zhang, Yi

Zhang, Yong

Zhang, Yongping

Zhang, Yudong

Zhang, Yushu

Zhao, Jichao

Zhao, Junming

Zhao, Lihao

Zhao, Xiaopeng

Zhong, Mingjun

Zhou, Jinjia

Zhou, Li

Zhou, Nan-Run

Zhu, Guohun

Zhu, Linhong

Ziemke, Tom

Zimborás, Zoltán

Zimdahl, Winfried

Zimparov, Ventsislav

Zmeskal, Oldrich

Zusai, Dai

(C) 2017 by the authors; licensee MDPI, Basel, Switzerland. This article is an open access article distributed under the terms and conditions of the Creative Commons Attribution (CC-BY) license (http://creativecommons.org/licenses/by/4.0/). 